R. Fowler and G. Röhrle

Nagoya Math. J.

Vol. 190 (2008), 105-128

\title{
ON COCHARACTERS ASSOCIATED TO NILPOTENT ELEMENTS OF REDUCTIVE GROUPS
}

\author{
RUSSELL FOWLER AND GERHARD RÖHRLE
}

\begin{abstract}
Let $G$ be a connected reductive linear algebraic group defined over an algebraically closed field of characteristic $p$. Assume that $p$ is good for $G$. In this note we consider particular classes of connected reductive subgroups $H$ of $G$ and show that the cocharacters of $H$ that are associated to a given nilpotent element $e$ in the Lie algebra of $H$ are precisely the cocharacters of $G$ associated to $e$ that take values in $H$. In particular, we show that this is the case provided $H$ is a connected reductive subgroup of $G$ of maximal rank; this answers a question posed by J. C. Jantzen.
\end{abstract}

\section{$\S 1$. Introduction}

Let $G$ be a connected reductive linear algebraic group defined over some algebraically closed field $k$, let $\mathfrak{g}$ be its Lie algebra and $\mathcal{N}$ be the nilpotent cone of $\mathfrak{g}$. The Jacobson-Morozov Theorem allows one to associate an $\mathfrak{s l}(2)$ triple to any given non-zero nilpotent element in $\mathcal{N}$ in characteristic zero or large positive characteristic. This is an indispensable tool in the DynkinKostant classification of the nilpotent orbits in characteristic zero as well as in the Bala-Carter classification of unipotent conjugacy classes of $G$ in large prime characteristic, see $[5, \S 5.9]$. In good characteristic there is a replacement for $\mathfrak{s l}(2)$-triples, so called associated cocharacters; see Definition 2.9 below. These cocharacters have become important tools in the classification theory of unipotent and nilpotent classes of reductive algebraic groups in good characteristic, see for instance [7, §5], [10], [12], [15], and [16] for more details.

In $[7, \S 5.6]$, J. C. Jantzen studies the behaviour of cocharacters associated to nilpotent elements under elementary operations of algebraic groups such as passing to derived subgroups, taking direct products and isogenies of reductive groups. While these cocharacters also behave well with respect to inclusions of reductive subgroups in characteristic zero, this is not the

Received December 12, 2006.

2000 Mathematics Subject Classification: Primary 20G15, 14L30; Secondary 17B50. 
case in general in positive characteristic, $[7, \S 5.12]$; given that they serve as a replacement for $\mathfrak{s l}(2)$-triples, this is somewhat surprising.

More precisely, in [7, Claim 5.12], Jantzen showed that if char $k=0$ and $H$ is a connected reductive subgroup of $G$ with Lie algebra $\mathfrak{h} \subseteq \mathfrak{g}$, then

$(\dagger)$ the cocharacters of $H$ associated to $e \in \mathfrak{h} \cap \mathcal{N}$ are precisely the cocharacters of $G$ associated to $e$ that take values in $H$.

Jantzen continues to give an example in $[7, \S 5.12]$ which shows that $(\dagger)$ fails in general in positive characteristic, even when char $k$ is good for $G$.

G. McNinch pointed out that this failure ultimately stems from the fact that the representations of $H$ are not semisimple in general in positive characteristic, as is the case in Jantzen's counterexample. The following construction due to McNinch shows that $(\dagger)$ fails generically: Let $H$ be a connected reductive group whose Coxeter number satisfies $h<p=$ char $k$. Let $K \leq H$ be a principal $\mathrm{SL}_{2}$-subgroup of $H$ and let $V$ be a faithful representation of $H$ for which there is a $K$-composition factor of the restriction of $V$ to $K$ having a non-restricted highest weight. See [11] for a definition, existence and uniqueness up to conjugacy of principal $\mathrm{SL}_{2}$-subgroups, see also [20]. A maximal torus of $K$ determines a cocharacter $\lambda$ of $H$ associated to some nilpotent element $e$ of Lie $K$. However, viewed as a cocharacter of $\mathrm{GL}(V), \lambda$ is not associated to $e$. In this sense, $(\dagger)$ fails for $H \leq \mathrm{GL}(V)$ for most representations $V$ of $H$.

Nevertheless, a calculation of Jantzen shows that for $G$ of classical type and char $k$ a good prime for $G,(\dagger)$ does hold provided the subgroup $H$ of $G$ is of maximal rank, see $[7, \S 5.12]$. In this note we give a general proof showing that this is indeed always the case irrespective of the type of $G$ :

THEOREM 1.1. Suppose char $k$ is good for $G$. Let $H$ be a connected reductive subgroup of $G$ of maximal rank. Then $(\dagger)$ holds.

This answers a question posed by J. C. Jantzen, see $[7, \S 5.12]$. We then extend this result to the case when $H$ is a regular reductive subgroup of $G$, see Theorem 3.29.

In Theorem 3.18 we show that $(\dagger)$ holds provided there is at least one cocharacter of $G$ that is associated to $e$ and lies in $H$. In our principal result we show that this is the case for a special class of subgroups:

THEOREM 1.2. Let $G$ be a connected reductive algebraic group. Suppose that char $k$ is a good prime for $G$. Let $S$ be a linearly reductive group acting on $G$ by automorphisms and set $H=C_{G}(S)^{\circ}$. Then $(\dagger)$ holds. 
Theorem 1.2 is a consequence of Theorem 3.18 and Lemma 3.20. Our proof uses ideas due to R. W. Richardson that he used in order to show that any $S$-stable parabolic subgroup of $G$ admits an $S$-stable Levi subgroup in [17, Prop. 6.1, §6.2, §6.3].

In turn Theorem 1.1 is a direct consequence of Theorem 1.2.

In Subsection 3.1 we consider arbitrary connected reductive subgroups $H$ of $G$ and give criteria for a given nilpotent element $e \in \mathfrak{h}$ that will ensure $(\dagger)$ to hold. For instance, in Lemma 3.1 we prove that this is the case provided $e \in \mathfrak{h}$ is distinguished in $\mathfrak{g}$. In another result, Theorem 3.14, we show that $(\dagger)$ holds given that the ranks of the centralizers of $e$ in $H$ and $G$ coincide.

We assume throughout that char $k$ is good for $G$. Then there exists a $G$ equivariant homeomorphism $\mathcal{N} \rightarrow \mathcal{U}$ between the nilpotent cone $\mathcal{N}$ of $\mathfrak{g}$ and the unipotent variety $\mathcal{U}$ of $G$. Such a map is called a Springer isomorphism, see [25, III, 3.12] and [1, Cor. 9.3.4]. By means of such a map, all the results below admit analogues for associated cocharacters of unipotent elements in G.

For general results on algebraic groups we cite Borel's book [2], and for basic results on cocharacters associated to nilpotent elements, we refer the reader to Jantzen's monograph [7, Ch. 5] and the articles by McNinchSommers [12] and Premet [16].

\section{$\S 2$. Preliminaries}

\subsection{Notation}

Throughout, $G$ is a connected reductive algebraic group defined over an algebraically closed field $k$ and $p=$ char $k$ is a good prime for $G$, although many results hold without this assumption. We denote the Lie algebra of $G$ by Lie $G$ or by $\mathfrak{g}$; likewise for closed subgroups of $G$. For $e \in \mathfrak{g}$ and $g \in G$ we denote the adjoint action of $g$ on $e$ by $\operatorname{Ad}(g) e$. The centralizers of $e$ in $G$ and $\mathfrak{g}$ are $C_{G}(e)=\{g \in G \mid \operatorname{Ad}(g) e=e\}$ and $\mathfrak{c}_{\mathfrak{g}}(e)=\{x \in \mathfrak{g} \mid[x, e]=0\}$, respectively. We write $Z(G)$ for the centre of $G$.

Let $H$ be a closed subgroup of $G$. We write $H^{\circ}$ for the identity component of $H$ and $C_{G}(H)=\left\{g \in G \mid g h g^{-1}=h\right.$ for all $\left.h \in H\right\}$ for the centralizer of $H$ in $G$. The normalizer of $H$ in $G$ is $N_{G}(H)=\left\{g \in G \mid g H^{-1}=H\right\}$. The derived subgroup of $H$ is denoted by $\mathcal{D} H$ and we write rank $H$ for the dimension of a maximal torus of $H$. The unipotent radical of $H$ is denoted by $R_{u}(H)$. A Levi subgroup of $H$ is a complement to $R_{u}(H)$ in $H,[2$, Defn. 11.22]; in contrast to loc. cit., we do not require $H$ to be connected; 
we also refer to the semi-direct product of a Levi subgroup of $H$ and $R_{u}(H)$ as a Levi decomposition of $H$.

By a Levi subgroup of $G$ we mean a Levi subgroup of a parabolic subgroup of $G$. Let $S$ be a torus of $G$. Then $C_{G}(S)$ is a Levi subgroup of $G$, [2, Thm. 20.4]. Note that $C_{G}(S)$ is connected, [2, Cor. 11.12]. Moreover, by $\left[2\right.$, Prop. 8.18] there exists an element $s \in S$ such that $C_{G}(s)=C_{G}(S)$. Conversely, every Levi subgroup of $G$ is of this form, e.g., see Lemma 2.6(ii).

Let $Y(G)=\operatorname{Hom}\left(k^{*}, G\right)$ denote the set of cocharacters of $G$. For $\mu \in$ $Y(G)$ we write $C_{G}(\mu)$ for $C_{G}\left(\mu\left(k^{*}\right)\right)$. For $\mu \in Y(G)$ and $g \in G$ we define the conjugate cocharacter $g \cdot \mu \in Y(G)$ by $(g \cdot \mu)(t)=g \mu(t) g^{-1}$; this gives a left action of $G$ on $Y(G)$. For $H$ a (connected) reductive subgroup of $G$, let $Y(H)=\operatorname{Hom}\left(k^{*}, H\right)$ denote the set of cocharacters of $H$. There is an obvious inclusion $Y(H) \subseteq Y(G)$.

If $S$ is a linear algebraic group acting on $G$ by automorphisms, then we say that $G$ is an $S$-group; we write $s \cdot g$ for the action of $s \in S$ on $g \in G$. The subgroup of $G$ consisting of the $S$-fixed points is denoted by $C_{G}(S)=\{g \in G \mid s \cdot g=g$ for all $s \in S\}$. If $G$ is an $S$-group, then $S$ acts naturally by means of Lie algebra automorphisms on $\mathfrak{g}$. By abuse of notation, we simply denote the action of $S$ on $\mathfrak{g}$ by $s \cdot e$ for $s \in S$ and $e \in \mathfrak{g}$. We denote the subalgebra of $\mathfrak{g}$ consisting of the $S$-fixed points for the induced action on $\mathfrak{g}$ by $\mathfrak{c}_{\mathfrak{g}}(S)=\{e \in \mathfrak{g} \mid s \cdot e=e$ for all $s \in S\}$. Also, $S$ acts on $Y(G)$ by acting on the image of a cocharacter in $G:(s \cdot \lambda)(t)=s \cdot \lambda(t)$ for $s \in S, \lambda \in Y(G)$, and $t \in k^{*}$.

More generally, if $S$ and the $S$-group $G$ both act morphically on an algebraic variety $X$, then, following [17, (2.1)], the actions of $G$ and $S$ are said to be compatible, provided

$$
s \cdot(g \cdot x)=(s \cdot g) \cdot(s \cdot x)
$$

for all $s \in S, g \in G$ and $x \in X$. This is the unique action that defines a morphic action of the semidirect product of $G$ and $S$ on $X$ which extends the actions of both $G$ and $S$ on $X$, see [17, §2]. All actions by an $S$-group $G$ together with $S$ considered in this paper are compatible in the sense of (2.1).

Let $T$ be a maximal torus of $G$. Let $\Psi=\Psi(G, T)$ denote the set of roots of $G$ with respect to $T$. Fix a Borel subgroup $B$ of $G$ containing $T$ and let $\Pi=\Pi(G, T)$ be the set of simple roots of $\Psi$ defined by $B$. Then $\Psi^{+}=\Psi(B)$ is the set of positive roots of $G$. For $\beta \in \Psi^{+}$write $\beta=\sum_{\alpha \in \Pi} c_{\alpha \beta} \alpha$ with 
$c_{\alpha \beta} \in \mathbb{N}_{0}$. A prime $p$ is said to be $\operatorname{good}$ for $G$ if it does not divide $c_{\alpha \beta}$ for any $\alpha$ and $\beta,[25$, Defn. 4.1].

\subsection{Linearly reductive groups}

We refer to Richardson's article [17] for information on centralizers of the action of linearly reductive groups on connected reductive groups. Recall that a linear algebraic group $S$, not necessarily connected, is said to be linearly reductive if every rational representation of $S$ is semisimple. It is well known that in characteristic zero, $S$ is linearly reductive if and only if $S^{\circ}$ is reductive. In characteristic $p>0, S$ is linearly reductive if and only if every element of $S$ is semisimple if and only if $S^{\circ}$ is a torus and $\left|S / S^{\circ}\right|$ is coprime to $p$, see $[14, \S 4$, Thm. 2]. In particular, a torus is linearly reductive.

In the sequel we require some fundamental results concerning centralizers of linearly reductive groups acting on connected reductive groups; the following facts are [17, Lem. 4.1, Prop. 10.1.5].

Proposition 2.2. Let $G$ be a connected reductive algebraic group and $S$ a linearly reductive algebraic group acting on $G$ so that $G$ is an $S$-group. Then we have

(i) $C_{G}(S)^{\circ}$ is reductive;

(ii) $\operatorname{Lie} C_{G}(S)=\mathfrak{c}_{\mathfrak{g}}(S)$.

The following result is due to R. W. Richardson, cf. [17, Prop. 6.1].

Proposition 2.3. Let $G$ be a connected reductive algebraic group and $S$ a linearly reductive algebraic group acting on $G$ so that $G$ is an $S$-group. Let $K$ be a (not necessarily connected) closed $S$-stable subgroup of $G$. Suppose that $K$ admits a Levi decomposition such that $R_{u}(K)$ acts simply transitively on the set of all Levi subgroups of $K$. Then $K$ admits an $S$-stable Levi subgroup.

Proof. Although [17, Prop. 6.1] is only stated for parabolic subgroups of $G$, Richardson's proof applies in this slightly more general setting mutatis mutandis. The given conditions on the Levi subgroups of $K$ are precisely the relevant properties of the set of Levi subgroups of a parabolic subgroup of $G$ in Richardson's proof, see $[17, \S 6.3]$. In addition, a general result on the vanishing of the (non-commutative) cohomology group $H^{1}\left(S, R_{u}(K)\right)$ is needed; this is proved by Richardson in [17, Lem. 6.2.6]. 
Remark 2.4. If $S$ is a subgroup of $K$ in Proposition 2.3, then the conclusion is that there exists a Levi subgroup of $K$ containing $S$, see Jantzen's generalization [7, Lem. 11.24] of Mostow's theorem [13, Thm. 7.1]. In that case the assumption that $R_{u}(K)$ acts simply transitively on the set of all Levi subgroups of $K$ is not necessary.

The essence of the arguments in the proofs of [7, Lem. 11.24], [13, Thm. 7.1], and [17, Lem. 6.2.6] is the vanishing of the cohomology $H^{1}(F, U)$, where $F$ is a finite group whose order is coprime to $p$ and $U$ is a (finite) unipotent group.

Remark 2.5. Let $\sigma$ be a semisimple automorphism of $G,[23, \S 7]$. Then there is an embedding $G \leq \mathrm{GL}_{n}$ of algebraic groups for some $n$ such that $\sigma$ is given by conjugation by a semisimple element, say $s$, in $\mathrm{GL}_{n}$. Thus $s$ belongs to some maximal torus of $\mathrm{GL}_{n}$, so the algebraic subgroup $S$ of $\mathrm{GL}_{n}$ generated by $s$ consists of semisimple elements. Thus $S$ is linearly reductive, by $[14, \S 4$, Thm. 2]. Clearly, $S$ depends on the choice of the ambient group $\mathrm{GL}_{n}$, but the fact that $S$ is linearly reductive does not.

\subsection{Regular reductive subgroups}

Let $H$ be a closed (not necessarily connected) subgroup of $G$ normalized by some maximal torus $T$ of $G$; that is, a regular subgroup of $G$ (reductive regular subgroups are often also referred to as subsystem subgroups in the literature). In this case the root spaces of $\mathfrak{h}$ relative to $T$ are also root spaces of $\mathfrak{g}$ relative to $T$, and the set of roots of $H$ with respect to $T$, $\Psi(H)=\Psi(H, T)=\left\{\alpha \in \Psi \mid \mathfrak{g}_{\alpha} \subseteq \mathfrak{h}\right\}$, is a subset of $\Psi$, where $\mathfrak{g}_{\alpha}$ denotes the root space in $\mathfrak{g}$ corresponding to $\alpha$. If char $k$ does not divide any of the structure constants of the Chevalley commutator relations of $G$, then $\Psi(H)$ is closed under addition. In particular, this is the case when char $k$ is a good prime for $G$. If $H$ is reductive and regular, then $\Psi(H)$ is a semisimple subsystem of $\Psi$.

Recall that for $s \in G$ semisimple, $H=C_{G}(s)^{\circ}$ is called a pseudo-Levi subgroup of $G$, cf. $[12, \S 6]$. Since $s$ is contained in a maximal torus $T$ of $G$, it follows that $H$ is regular of maximal rank. Moreover, $\Psi(H)=\Psi(H, T)=$ $\{\alpha \in \Psi(G, T) \mid \alpha(s)=0\}$.

\subsection{Kempf-Rousseau theory}

Let $G$ be a reductive group acting on an affine variety $X$. For $x \in X$ let $G \cdot x$ denote the $G$-orbit of $x$ in $X$ and $C_{G}(x)$ the stabilizer of $x$ in $G$. Let $\phi: k^{*} \rightarrow X$ be a morphism of algebraic varieties. We say that $\lim _{t \rightarrow 0} \phi(t)$ 
exists if there exists a morphism $\widehat{\phi}: k \rightarrow X$ (necessarily unique) whose restriction to $k^{*}$ is $\phi$; if this limit exists, then we set $\lim _{t \rightarrow 0} \phi(t)=\widehat{\phi}(0)$.

Recall the characterization of parabolic subgroups of $G$ in terms of cocharacters of $G$, e.g. see [22, Prop. 8.4.5].

LEMma 2.6. Given a parabolic subgroup $P$ of $G$ and any Levi subgroup $L$ of $P$, there exists $\lambda \in Y(G)$ such that the following hold:

(i) $P=P_{\lambda}:=\left\{g \in G \mid \lim _{t \rightarrow 0} \lambda(t) g \lambda(t)^{-1}\right.$ exists $\}$.

(ii) $L=L_{\lambda}:=C_{G}(\lambda)$.

(iii) $R_{u}(P)=\left\{g \in G \mid \lim _{t \rightarrow 0} \lambda(t) g \lambda(t)^{-1}=1\right\}$.

Conversely, given any $\lambda \in Y(G)$ the subset $P_{\lambda}$ defined as in part (i) is a parabolic subgroup of $G$ and $L_{\lambda}$ is a Levi subgroup of $P_{\lambda}$.

Let $G$ act morphically on the affine algebraic variety $X$. Let $x \in X$ and let $C$ be the unique closed orbit in the closure of $G \cdot x$. Then there exists a subset $\Omega(x)$ of $Y(G)$ consisting of so called optimal cocharacters $\lambda$ such that $\lim _{t \rightarrow 0} \lambda(t) \cdot x$ belongs to $C,[8],[18]$; see $[10, \S 3]$ or $[16, \S 2.2]$ for the relevant parts of the theory; see also Slodowy's survey article [21]. We record the crucial points of this theory.

THEOREM 2.7. Let $G$ act morphically on the affine algebraic variety $X$. Let $x \in X$ and let $\Omega(x) \subseteq Y(G)$ be the optimal class of cocharacters defined by $x$. Then the following hold:

(i) $\Omega(x) \neq \varnothing$ and there exists an optimal parabolic subgroup $P=P(x)$ of $G$ so that $P=P_{\lambda}$ for every $\lambda \in \Omega(x)$.

(ii) $\Omega(x)$ is a single P-orbit.

(iii) For every $g \in G$, we have $\Omega(g \cdot x)=g \cdot \Omega(x)$ and $P(g \cdot x)=g P(x) g^{-1}$. In particular, $C_{G}(x) \leq P$.

\subsection{Cocharacters associated to nilpotent elements}

Recall that any cocharacter $\lambda \in Y(G)$ of $G$ affords a $\mathbb{Z}$-grading

$$
\mathfrak{g}=\bigoplus_{j \in \mathbb{Z}} \mathfrak{g}(j, \lambda)
$$

of $\mathfrak{g}$, where

$$
\mathfrak{g}(j, \lambda):=\left\{e \in \mathfrak{g} \mid \operatorname{Ad}(\lambda(t)) e=t^{j} e \text { for every } t \in k^{*}\right\},
$$


cf. $[5, \S 5.5]$ or $[7, \S 5.1]$. We recall the relevant concepts of distinguished nilpotent elements and of cocharacters associated to a nilpotent element following [7, §4.1, §5.3].

DeFINITION 2.8. Let $H \leq G$ be a closed connected reductive subgroup of $G$ and $e \in \mathcal{N} \cap \mathfrak{h}$. We call $e$ distinguished in $\mathfrak{h}$ if each torus contained in $C_{H}(e)$ is contained in the centre of $H$.

Definition 2.9. Let $e \in \mathcal{N}$. A cocharacter $\lambda: k^{*} \rightarrow G$ of $G$ is called associated to $e$ provided $e \in \mathfrak{g}(2, \lambda)$ and there exists a Levi subgroup $L$ of $G$ such that $e$ is distinguished nilpotent in Lie $L$ and $\lambda\left(k^{*}\right) \leq \mathcal{D} L$.

Remarks 2.10. Let $e \in \mathcal{N}$ and let $\lambda \in Y(G)$ that is associated to $e$.

(i) For $g \in C_{G}(e)$ the conjugate cocharacter $g \cdot \lambda$ is also associated to $e$, cf. $[7, \S 5.3]$. Proposition 2.11(ii) gives a converse to this property.

(ii) Let $S$ be a maximal torus of $C_{G}(e)$. Then $e$ is distinguished in $\mathfrak{c}_{\mathfrak{g}}(S)=$ Lie $C_{G}(S)$, for $S$ is the unique maximal torus of $C_{C_{G}(S)}(e)$. Proposition 2.11(iii) gives a converse to this.

We require some basic facts about cocharacters associated to nilpotent elements; the following results are [7, Rem. 4.7; Lem. 5.3], see also [16, Prop. 2.5].

Proposition 2.11. Let $e \in \mathcal{N}$.

(i) Suppose char $k$ is good for $G$. Then cocharacters of $G$ associated to $e$ exist.

(ii) Any two cocharacters of $G$ associated to e are conjugate by an element of $C_{G}(e)^{\circ}$.

(iii) If $L$ is a Levi subgroup of $G$ with e distinguished in Lie $L$, then the connected centre of $L$ is a maximal torus of $C_{G}(e)^{\circ}$.

Remark 2.12. The Dynkin-Kostant classification theory giving a bijection between nilpotent $G$-classes and $G$-conjugacy classes of $\mathfrak{s l}(2)$-triples is also valid in large positive characteristic (more precisely, when char $k>$ $3(h-1)$, where $h$ denotes the Coxeter number of $G$, cf. $[5, \S \S 5.3-5.6])$. For $e \in \mathcal{N}$, the cocharacters of $G$ constructed from the semisimple elements of $\mathfrak{s l}(2)$-triples containing $e(\mathrm{cf} .[5, \S 5.5])$ are all associated to $e$ in the sense of Definition 2.9, see [7, Rem. 5.5]. 
Let $e \in \mathcal{N}$. In $[16, \S 2.4]$, A. Premet explicitly defines a cocharacter of $G$ which is associated to $e$, thanks to [16, Prop. 2.5]. Moreover, in [16, Thm. 2.3], Premet shows that each of these associated cocharacters belongs to the optimal class determined by $e$. Premet proves this under the so called standard hypotheses on $G$, see $[7, \S 2.9]$. These restrictions were subsequently removed by G. McNinch in [10, Prop. 16] so that this fact holds for any reductive $G$ in good characteristic. It thus follows from [10, Prop. 16], Theorem 2.7, and Proposition 2.11(ii) that all the cocharacters of $G$ associated to $e \in \mathcal{N}$ belong to the optimal class $\Omega(e)$ defined by $e$; see also [10, Prop. 18, Thm. 21]. This motivates and justifies the following notation which we frequently use in the sequel.

Definition 2.13. Let $e \in \mathcal{N}$. Then we define

$$
\Omega^{a}(e):=\{\lambda \in Y(G) \mid \lambda \text { is associated to } e\} \subseteq \Omega(e) .
$$

Further, we sometimes write $\Omega_{G}^{a}(e)$ for $\Omega^{a}(e)$, to indicate that this is a set of cocharacters of $G$, and if $H$ is a reductive subgroup of $G$ with $e \in \mathfrak{h}$ nilpotent we also write $\Omega_{H}^{a}(e)$ to denote the cocharacters of $H$ that are associated to $e$.

For $H$ a connected reductive subgroup of $G$ and $e \in \mathfrak{h} \cap \mathcal{N}$, in the notation of Definition 2.13, property $(\dagger)$ from page 106 becomes the equality

$$
\Omega_{H}^{a}(e)=\Omega_{G}^{a}(e) \cap Y(H) .
$$

Let $e \in \mathcal{N}$ and let $\lambda \in \Omega^{a}(e)$. Let $P=P(e)$ be the canonical parabolic subgroup defined by $e$. Then $P=C_{G}(\lambda) R_{u}(P)$ is a Levi decomposition of $P$. Thanks to Theorem 2.7(iii), $C_{G}(e) \leq P$. Following $[7, \S 5.10]$ and $[16$, $\S 2.4]$, we define the subgroups

$$
C_{G}(e, \lambda):=C_{G}(e) \cap C_{G}(\lambda) \quad \text { and } \quad R_{e}:=C_{G}(e) \cap R_{u}(P)
$$

of $C_{G}(e)$.

In view of [16, Prop. 2.5], our next result is [16, Thm. 2.3(iii)], see also [7, Prop. 5.10, Prop. 5.11].

Proposition 2.15. Suppose that char $k$ is good for $G$. Let $e \in \mathcal{N}$ and let $\lambda \in \Omega^{a}(e)$. Then $C_{G}(e)$ is the semidirect product of $C_{G}(e, \lambda)$ and $R_{e}$, where $C_{G}(e, \lambda)^{\circ}$ is reductive and $R_{e}=R_{u}\left(C_{G}(e)\right)$. 
Proposition 2.15 says that $C_{G}(e)=C_{G}(e, \lambda) R_{e}$ is a Levi decomposition of $C_{G}(e)$, so that different choices of associated cocharacters in $\Omega^{a}(e)$ give conjugate Levi subgroups $C_{G}(e, \lambda)$ of $C_{G}(e)$, by Proposition 2.11(ii). Our next result now readily follows from these two propositions.

Corollary 2.16. Suppose that char $k$ is good for $G$. Let $e \in \mathcal{N}$. Then $R_{e}$ acts simply transitively on $\Omega^{a}(e)$.

Remark 2.17. It follows from Propositions 2.11, 2.15 and Corollary 2.16 that the map $\lambda \mapsto C_{G}(e, \lambda)$ is a bijection between $\Omega^{a}(e)$ and the set of Levi subgroups of $C_{G}(e)$.

Let $e \in \mathfrak{g}$ be nilpotent and let $\lambda \in \Omega^{a}(e)$. It follows readily from Definition 2.9 that $C_{G}(e)$ is normalized by $\lambda\left(k^{*}\right)$, thus we may define the subgroup

$$
N_{e}:=\lambda\left(k^{*}\right) C_{G}(e)
$$

of $G$, cf. [7, $\S 5.3(2)]$. According to Proposition 2.11(ii), $N_{e}$ does not depend on the choice of $\lambda \in \Omega^{a}(e)$; this is also apparent, since $N_{e}=\{g \in G \mid g \cdot k e=$ $k e\}$, see $[7, \S 2.10(2)]$. Clearly, $\lambda\left(k^{*}\right)$ also normalizes $R_{e}=R_{u}\left(C_{G}(e)\right)$. Thus we may define the subgroup

$$
Q_{e}:=\lambda\left(k^{*}\right) R_{e}
$$

of $N_{e}$. By Corollary 2.16, equally $Q_{e}$ does not depend on the choice of $\lambda$ in $\Omega^{a}(e)$.

Let $H$ be a connected reductive subgroup of $G$. Since the nilpotent cone of Lie $\mathcal{D} H$ is a closed subvariety of the nilpotent variety of $\mathfrak{h}$, and both are irreducible of the same dimension, we have $\mathfrak{h} \cap \mathcal{N}=\operatorname{Lie}(\mathcal{D} H) \cap \mathcal{N}$.

LEMma 2.19. Let $H$ be a connected reductive subgroup of $G$. Let $e \in$ $\mathfrak{h} \cap \mathcal{N}=\operatorname{Lie}(\mathcal{D} H) \cap \mathcal{N}$ be nilpotent. Then the cocharacters of $\mathcal{D} H$ associated to $e$ are precisely the cocharacters of $H$ associated to $e$.

Proof. Assume that $\lambda$ is a cocharacter of $\mathcal{D} H$ associated to $e$. Note that $H=Z(H)^{\circ} \mathcal{D} H$. Let $L^{\prime}=C_{\mathcal{D} H}\left(S^{\prime}\right)$ be a Levi subgroup of $\mathcal{D} H$ satisfying the conditions of Definition 2.9, where $S^{\prime}$ is a maximal torus of $C_{\mathcal{D} H}(e)$. Then $S=Z(H)^{\circ} S^{\prime}$ is a maximal torus of $C_{H}(e)$ containing $S^{\prime}$. Set $L=$ $C_{H}(S)=Z(H)^{\circ} L^{\prime}$. It follows easily that $e$ is distinguished in Lie $L$. Further, $\lambda\left(k^{*}\right) \leq \mathcal{D}\left(L^{\prime}\right)=\mathcal{D} L$, and so $\lambda$ is associated to $e$, viewed as a cocharacter of $H$.

The reverse implication of the lemma is shown in $[7, \S 5.6]$. 


\section{$\S 3$. Cocharacters associated to nilpotent elements of reductive subgroups}

We maintain the notation and assumption of the previous sections. In particular, $G$ is a connected reductive algebraic group defined over an algebraically closed field $k$, char $k$ is a good prime for $G$, and $H$ is a closed connected reductive subgroup of $G$.

\subsection{Local conditions}

In this subsection we study conditions on a given nilpotent element $e$ in $\mathfrak{h}$ (or nilpotent $H$-class in $\mathfrak{h}$ ) that ensure $(\dagger)$ from page 106 holds for $e$ without further assumptions on $H$ itself. Firstly we consider nilpotent elements $e \in \mathfrak{h}$ that are distinguished in $\mathfrak{g}$, Lemma 3.1, and secondly we study the case when the centralizers in $H$ and $G$ of $e$ have the same rank, Theorem 3.14.

Lemma 3.1. Suppose that $e \in \mathfrak{h}$ is nilpotent and distinguished in $\mathfrak{g}$. Then $e$ is distinguished in $\mathfrak{h}$ and $\Omega_{H}^{a}(e)=\Omega_{G}^{a}(e) \cap Y(H)$.

Proof. Let $S$ be a torus of $C_{H}(e)$. Since $e$ is distinguished in $\mathfrak{g}$ and $C_{H}(e) \leq C_{G}(e)$, we have $S \leq Z(G)$. So $S \leq Z(G) \cap H \leq Z(H)$ and thus $e$ is distinguished in $\mathfrak{h}$.

First let $\lambda \in \Omega_{H}^{a}(e)$. Then $e \in \mathfrak{h}(2, \lambda) \subseteq \mathfrak{g}(2, \lambda)$. By Lemma 2.19, we have $\lambda\left(k^{*}\right) \leq \mathcal{D} H \leq \mathcal{D} G$, and so $\lambda \in \Omega_{G}^{a}(e)$, as $e$ is distinguished in $\mathfrak{g}$.

Conversely, let $\lambda \in \Omega_{G}^{a}(e)$ with $\lambda\left(k^{*}\right) \leq H$. Since $e$ is distinguished in $\mathfrak{h}$, and since $e \in \mathfrak{h} \cap \mathfrak{g}(2, \lambda)=\mathfrak{h}(2, \lambda)$, it suffices to show that $\lambda\left(k^{*}\right) \leq$ $\mathcal{D} H$. Since $e$ is distinguished in $\mathfrak{g}$ and $Z(H)^{\circ} \leq C_{H}(e) \leq C_{G}(e)$, we have $Z(H)^{\circ} \leq Z(G)^{\circ} \cap H$. Note that $\mathcal{D} H \leq \mathcal{D} G \cap H \leq H$. Since $H$ is reductive, we have $H=Z(H)^{\circ} \mathcal{D} H$, so that $\mathcal{D} G \cap H=A \mathcal{D} H$, where $A$ is a subgroup of $Z(H)^{\circ}$. By assumption, $\lambda\left(k^{*}\right) \leq \mathcal{D} G \cap H$. Since $\lambda\left(k^{*}\right)$ is connected, we have $\lambda\left(k^{*}\right) \leq(\mathcal{D} G \cap H)^{\circ}=A^{\circ} \mathcal{D} H$. Because $A^{\circ} \leq Z(H)^{\circ} \leq Z(G)^{\circ} \cap H$ and $A^{\circ} \leq \mathcal{D} G \cap H$, we have $A^{\circ} \leq \mathcal{D} G \cap Z(G)^{\circ}$, and so $A^{\circ}$ is trivial. Consequently, $\lambda\left(k^{*}\right) \leq \mathcal{D} H$, as desired.

We give an example for Lemma 3.1.

ExAmple 3.2. Let $G$ be simple of type $E_{6}$ and let $H$ be the fixed point subgroup of the non-trivial graph automorphism of $G$; so that $H$ is of type $F_{4}$. Let $C^{\prime}$ be the nilpotent $H$-class with Bala-Carter label $F_{4}\left(a_{2}\right)$ and let $C$ be the nilpotent $G$-class with Bala-Carter label $E_{6}\left(a_{3}\right)$. According to $[9$, 
Table A], we have $C^{\prime} \subset C$. Note that each of these classes is distinguished, see $[5, \S 5.9]$. Thus Lemma 3.1 applies. Another example is given by the regular nilpotent class in $H$ which belongs to the regular $G$-class in $\mathcal{N}$; they are obviously both distinguished. Although the results in $[5, \S 5.9]$ and [9] concern unipotent classes in $G$, since $p$ is good for $G$, they equally apply to nilpotent classes in $\mathfrak{g}$.

Corollary 3.24 below implies that in this case the conclusion of Lemma 3.1 holds for any nilpotent class of $H$.

Lemma 3.3. Let $e \in \mathfrak{h}$ be nilpotent. Suppose that $\operatorname{rank} C_{G}(e)=$ $\operatorname{rank} C_{H}(e)$. Then $\Omega_{H}^{a}(e) \subseteq \Omega_{G}^{a}(e) \cap Y(H)$.

Proof. Let $\lambda \in \Omega_{H}^{a}(e)$. Since $e \in \mathfrak{h}(2, \lambda) \subseteq \mathfrak{g}(2, \lambda)$, it suffices to find a Levi subgroup $L$ of $G$ such that $e$ is distinguished in Lie $L$ and $\lambda\left(k^{*}\right) \leq \mathcal{D} L$. Let $M$ be a Levi subgroup of $H$ with the properties as in Definition 2.9. Thanks to Proposition 2.11(iii), we have $M=C_{H}(S)$, where $S$ is a maximal torus of $C_{H}(e)$. Since $C_{H}(e) \leq C_{G}(e)$ and by our hypothesis, $S$ is also a maximal torus of $C_{G}(e)$. Thus $e$ is distinguished in $\operatorname{Lie} C_{G}(S)$, cf. Remark 2.10(ii). Finally, since $\lambda\left(k^{*}\right) \leq \mathcal{D} C_{H}(S) \leq \mathcal{D} C_{G}(S)$, we see that $\lambda \in \Omega_{G}^{a}(e)$, as desired.

Let $e \in \mathfrak{g}$ be nilpotent. Let $\lambda$ be a cocharacter of $G$ associated to $e$. Define

$$
\begin{array}{r}
\Upsilon_{\lambda}(e)=\left\{S \leq C_{G}(e) \mid S \text { is a maximal torus of } C_{G}(e)\right. \\
\text { and } \left.\lambda\left(k^{*}\right) \leq \mathcal{D} C_{G}(S)\right\} .
\end{array}
$$

Note that by Proposition 2.11(iii), $\Upsilon_{\lambda}(e)$ is non-empty.

For our next result recall the definition of $C_{G}(e, \lambda)=C_{G}(e) \cap C_{G}(\lambda)$ from (2.14).

Lemma 3.5. Let $e \in \mathfrak{g}$ be nilpotent. Let $\lambda$ be a cocharacter of $G$ associated to $e$. Then $\Upsilon_{\lambda}(e)$ consists precisely of the maximal tori of $C_{G}(e, \lambda)$.

Proof. Let $S \in \Upsilon_{\lambda}(e)$. Then $S$ is a maximal torus of $C_{G}(e)$ and $\lambda\left(k^{*}\right) \leq \mathcal{D} C_{G}(S) \leq C_{G}(S)$. In particular, $S \leq C_{G}(\lambda)$ and so $S \leq C_{G}(e, \lambda)$. Thus $S$ is a maximal torus of $C_{G}(e, \lambda)$.

Conversely, let $S$ be a maximal torus of $C_{G}(e, \lambda)$. Then, by what we have just shown, $S$ is conjugate in $C_{G}(e, \lambda)$ to some $S^{\prime} \in \Upsilon_{\lambda}(e)$, and so 
in particular, $S$ is a maximal torus of $C_{G}(e)$. Let $g \in C_{G}(e, \lambda)$ so that $S=g S^{\prime} g^{-1}$. Since $\lambda\left(k^{*}\right) \leq \mathcal{D} C_{G}\left(S^{\prime}\right)$, we have $g \lambda\left(k^{*}\right) g^{-1} \leq g \mathcal{D} C_{G}\left(S^{\prime}\right) g^{-1}=$ $\mathcal{D} C_{G}(S)$. Finally, since $g \in C_{G}(\lambda)$, we obtain $g \lambda\left(k^{*}\right) g^{-1}=\lambda\left(k^{*}\right)$, and therefore, $S \in \Upsilon_{\lambda}(e)$, as desired.

Lemma 3.6. Let $e \in \mathfrak{h}$ be nilpotent. Let $\lambda \in \Omega_{G}^{a}(e)$ with $\lambda\left(k^{*}\right) \leq H$. Suppose there exists a maximal torus of $C_{H}(e)$ which is also a maximal torus of $C_{G}(e, \lambda)$. Then $\lambda \in \Omega_{H}^{a}(e)$.

Proof. Since $e \in \mathfrak{h} \cap \mathfrak{g}(2, \lambda)=\mathfrak{h}(2, \lambda)$, it suffices to find a Levi subgroup $M$ of $H$ such that $e$ is distinguished in Lie $M$ and $\lambda\left(k^{*}\right) \leq \mathcal{D} M$. Let $L$ be a Levi subgroup of $G$ such that $e$ is distinguished in Lie $L$ and $\lambda\left(k^{*}\right) \leq \mathcal{D} L$. By Proposition 2.11(iii), we have $L=C_{G}(S)$, where $S$ is a maximal torus of $C_{G}(e)$. By Remark 2.10(ii), $\lambda$ is a cocharacter of $C_{G}(S)$ that is associated to $e$ and $e$ is distinguished in $\mathfrak{c}_{\mathfrak{g}}(S)=\operatorname{Lie} C_{G}(S)$. By our hypothesis and Lemma 3.5, we may assume without loss of generality that $S$ is a maximal torus of $C_{H}(e)$. Then $S \leq H$ and so $C_{H}(S)$ is a Levi subgroup of $H$. Consequently, by Lemma 3.1 applied to $C_{H}(S) \leq C_{G}(S)$, we get that $\lambda$ is a cocharacter of $C_{H}(S)$ associated to $e$. Since $S$ is a maximal torus of $C_{H}(e)$ as well as of $C_{C_{H}(S)}(e)$, we can apply Lemma 3.3 to $C_{H}(S) \leq H$ and so $\lambda \in \Omega_{H}^{a}(e)$.

Let $e \in \mathfrak{h}$ be nilpotent and let $\lambda$ be a cocharacter of $G$ associated to $e$ with $\lambda\left(k^{*}\right) \leq H$. Let $P$ be the parabolic subgroup of $G$ defined by $\lambda$, i.e., $P=P_{\lambda}$, cf. Lemma 2.6(i). Since $\lambda$ is optimal for $e$, we have $P=P(e)$ is the optimal parabolic subgroup determined by $e$, cf. Theorem 2.7(i). Define $P_{H}=P \cap H$. Since $\lambda\left(k^{*}\right) \leq H$, we see $P_{H}$ is a parabolic subgroup of $H$. Also $P_{H}$ has Lie algebra $\bigoplus_{i>0} \mathfrak{h}(i, \lambda)$ and the unipotent radical $R_{u}\left(P_{H}\right)$ of $P_{H}$ is $R_{u}(P) \cap H$ and $C_{H}(\lambda)=C_{G}(\lambda) \cap H$ is a Levi subgroup of $P_{H}$. The unipotent radical of $P_{H}$ and the Levi subgroup $C_{H}(\lambda)$ have Lie algebras $\bigoplus_{i>0} \mathfrak{h}(i, \lambda)$ and $\mathfrak{h}(0, \lambda)$, respectively, see $[7, \S 5.1]$. Analogous to $(2.14)$ define the subgroups

$$
C_{H}(e, \lambda):=C_{H}(e) \cap C_{H}(\lambda) \quad \text { and } \quad U_{e}:=C_{H}(e) \cap R_{u}\left(P_{H}\right)
$$

of $C_{H}(e)$. Note that $C_{H}(e, \lambda) \cap U_{e}=\{1\}$, so $C_{H}(e, \lambda) U_{e}$ is a semidirect product of algebraic groups. Next we require the following facts, see $[7$, 
$\S 5.10]$. For all $i \in \mathbb{Z}$ we have

$$
\begin{aligned}
\operatorname{Ad}(x)(\mathfrak{h}(i, \lambda)) & =\mathfrak{h}(i, \lambda) \text { for all } x \in C_{H}(\lambda) \\
(\operatorname{Ad}(y)-1)(\mathfrak{h}(i, \lambda)) & \subseteq \bigoplus_{j>i} \mathfrak{h}(j, \lambda) \text { for all } y \in R_{u}\left(P_{H}\right) .
\end{aligned}
$$

LEMMA 3.10. With the notation introduced in (3.7), we have the following.

(i) $C_{H}(e) \leq P_{H}$.

(ii) $U_{e}$ is a normal connected unipotent subgroup of $C_{H}(e)$.

(iii) $C_{H}(e)$ is the semidirect product of $C_{H}(e, \lambda)$ and $U_{e}$.

Proof. (i) Let $x \in C_{H}(e)=C_{G}(e) \cap H$. Since $\lambda \in \Omega^{a}(e)$, we have $C_{G}(e) \subseteq P$, by Theorem 2.7(iii). Therefore, $x \in P \cap H=P_{H}$.

(ii) By definition, $U_{e}$ is unipotent. Let $x \in U_{e}$ and $y \in C_{H}(e)$. Now $x \in R_{u}\left(P_{H}\right)$ and $y \in P_{H}$. So $y x y^{-1} \in R_{u}\left(P_{H}\right)$, as $R_{u}\left(P_{H}\right)$ is normal in $P_{H}$. Also $x, y \in C_{H}(e)$ so $y x y^{-1} \in R_{u}\left(P_{H}\right) \cap C_{H}(e)=U_{e}$. Since $U_{e}$ is normalized by $\lambda\left(k^{*}\right)$, it is connected: For any $x \in U_{e}$ we have a morphism $\phi_{x}: k \rightarrow U_{e}$ given by $\phi_{x}(t)=\lambda(t) x \lambda(t)^{-1}$ for $t \in k^{*}$ and $\phi_{x}(0)=\lim _{t \rightarrow 0} \lambda(t) x \lambda(t)^{-1}=1$, cf. Lemma 2.6(iii). Thus for each $x \in U_{e}$ the image of $\phi_{x}$ is a connected subvariety of $U_{e}$ containing 1 and $x=\phi_{x}(1)$.

(iii) Let $z \in C_{H}(e)$. Thanks to part (i), we can write $z$ as $z=x y$ with $x \in C_{H}(\lambda)$ and $y \in R_{u}\left(P_{H}\right)$. Since $e \in \mathfrak{h}(2, \lambda)$, it follows from (3.9) that $\operatorname{Ad}(y)(e)=e+e^{\prime}$ with $e^{\prime} \in \bigoplus_{i>3} \mathfrak{h}(i, \lambda)$. Since $z \in C_{H}(e)$, we have $\operatorname{Ad}(x)\left(e+e^{\prime}\right)=e$. It follows that $\overline{\operatorname{Ad}}(x)\left(e^{\prime}\right)=0$, thanks to (3.8). As $\operatorname{Ad}(x): \mathfrak{h} \rightarrow \mathfrak{h}$ is a Lie algebra automorphism of $\mathfrak{h}$, we infer that $e^{\prime}=0$. Consequently, $y \in C_{H}(e) \cap R_{u}\left(P_{H}\right)$ and thus $x \in C_{H}(e) \cap C_{H}(\lambda)$. Therefore, $C_{H}(e)=C_{H}(e, \lambda) U_{e}$.

Remark 3.11. Despite the analogy between Proposition 2.15 and Lemma 3.10, the semidirect product $C_{H}(e)=C_{H}(e, \lambda) U_{e}$ need not be a Levi decomposition of $C_{H}(e)$; we cannot invoke Proposition 2.15, as we do not know whether $\lambda$ lies in $\Omega_{H}^{a}(e)$. It follows from Theorem 3.21 that this is the case for the particular class of subgroups $H$ of $G$ considered there.

Lemma 3.12. Let $e \in \mathfrak{h}$ be nilpotent. Suppose that $\operatorname{rank} C_{G}(e)=$ $\operatorname{rank} C_{H}(e)$. Let $\lambda \in \Omega_{G}^{a}(e) \cap Y(H)$. Then there exists a maximal torus of $C_{H}(e)$ which is also a maximal torus of $C_{G}(e, \lambda)$. 
Proof. It follows from Lemma 3.10(iii) that $\operatorname{rank} C_{H}(e)=\operatorname{rank}$ $C_{H}(e, \lambda)$. Obviously, we have $C_{H}(e, \lambda) \leq C_{G}(e, \lambda) \leq C_{G}(e)$. It follows from the assumption on the rank of the centralizers of $e$ in $H$ and in $G$ that a maximal torus of $C_{H}(e, \lambda)$, therefore of $C_{H}(e)$, is also a maximal torus of $C_{G}(e, \lambda)$.

Our next result is a consequence of Lemmas 3.6 and 3.12.

Proposition 3.13. Let $e \in \mathfrak{h}$ be nilpotent. Suppose that $\operatorname{rank} C_{G}(e)=$ $\operatorname{rank} C_{H}(e)$. Then $\Omega_{G}^{a}(e) \cap Y(H) \subseteq \Omega_{H}^{a}(e)$.

Our next result follows immediately from Lemma 3.3 and Proposition 3.13 .

Theorem 3.14. Let $e \in \mathfrak{h}$ be nilpotent. Suppose that $\operatorname{rank} C_{G}(e)=$ $\operatorname{rank} C_{H}(e)$. Then $\Omega_{H}^{a}(e)=\Omega_{G}^{a}(e) \cap Y(H)$.

We give an example for Theorem 3.14.

ExAmple 3.15. We return to the case of Example 3.2. Let $G$ be simple of type $E_{6}$ and let $H$ be the standard subgroup of $G$ of type $F_{4}$. Let $D^{\prime}$ be the nilpotent $H$-class with Bala-Carter label $\tilde{A}_{2}$ and let $D$ be the nilpotent $G$-class with Bala-Carter label $2 A_{2}$. According to [9, Table A], we have $D^{\prime} \subset D$. It is known that the reductive parts of the centralizers of these classes in $H$ and $G$ are of type $G_{2}$ in each case, see [5, Ch. 13]. In particular, the rank condition in Theorem 3.14 is satisfied for an element belonging to $D^{\prime}$. This also applies to the classes with Bala-Carter labels $C_{3}$ in $H$ and $A_{5}$ in $G$, here the reductive parts of the centralizers are of type $A_{1}$ in each case. There is one further pair of classes with the same property.

It follows from Corollary 3.24 below that in this example the conclusion of Theorem 3.14 holds for any nilpotent element of $\mathfrak{h}$ irrespective of the condition on the ranks of the respective centralizers.

See also Example 3.33 below for another application of Theorem 3.14.

\subsection{Global conditions}

We maintain the notation from the previous sections. In this subsection we study conditions on the subgroup $H$ of $G$ (rather than on a given nilpotent $H$-class in $\mathfrak{h}$ ) that ensure that $(\dagger)$ holds for all $e \in \mathcal{N} \cap \mathfrak{h}$.

Our first result shows that the reverse inclusion of $(\dagger)$ always holds in good characteristic without any restrictions on $H$. 
Proposition 3.16. Let $e \in \mathfrak{h}$ be nilpotent. Then $\Omega_{G}^{a}(e) \cap Y(H) \subseteq$ $\Omega_{H}^{a}(e)$.

Proof. Let $\lambda \in \Omega_{G}^{a}(e)$ with $\lambda\left(k^{*}\right) \leq H$. First assume that $e$ is distinguished in $\mathfrak{h}$. Since $e \in \mathfrak{h} \cap \mathfrak{g}(2, \lambda)=\mathfrak{h}(2, \lambda)$ and $e$ is distinguished in $\mathfrak{h}$, it suffices to show that $\lambda\left(k^{*}\right) \leq \mathcal{D} H$. Set $Z=Z(H)^{\circ}$. Then $Z \leq C_{H}(e, \lambda) \leq C_{G}(e, \lambda)$. Choose a maximal torus $S$ of $C_{G}(e, \lambda)$ so that $Z \leq S$. Thanks to Lemma 3.5, $S$ is a maximal torus of $C_{G}(e)$. Since $S \leq C_{G}(e, \lambda)$, we have $\lambda\left(k^{*}\right) \leq \mathcal{D} C_{G}(S)$, by Lemma 3.5. Also, as $Z \leq S$, we have $C_{G}(S) \leq C_{G}(Z)$. Thus $\lambda\left(k^{*}\right) \leq \mathcal{D} C_{G}(Z)$ and so $\lambda\left(k^{*}\right) \leq H \cap \mathcal{D} C_{G}(Z)$. As $H \leq C_{G}(Z)$, we get $\mathcal{D} H \leq H \cap \mathcal{D} C_{G}(Z) \leq H$. Since $H$ is reductive, we have $H=Z \mathcal{D} H$, so $H \cap \mathcal{D} C_{G}(Z)=A \mathcal{D} H$, where $A \leq Z$. As $\lambda\left(k^{*}\right)$ is connected, $\lambda\left(k^{*}\right) \leq A^{\circ} \mathcal{D} H$. Clearly, we have $A^{\circ} \leq A \mathcal{D} H=H \cap \mathcal{D} C_{G}(Z)$ and $A^{\circ} \leq Z$ so $A^{\circ} \leq Z \cap \mathcal{D} C_{G}(Z)$. Since $C_{G}(Z)$ is a connected reductive subgroup of $G$ and $Z$ is contained in the connected centre of $C_{G}(Z)$, it follows that $Z \cap \mathcal{D} C_{G}(Z)$ is finite. Thus $A^{\circ}$ is trivial and hence $\lambda\left(k^{*}\right) \leq \mathcal{D} H$, as desired.

Now we consider the general case where $e$ is not necessarily distinguished in $\mathfrak{h}$. Let $S$ be a maximal torus of $C_{H}(e, \lambda)$. By Lemma 3.10(iii), $S$ is then also a maximal torus of $C_{H}(e)$. Since $S \leq C_{G}(\lambda)$, we have $\lambda\left(k^{*}\right) \leq C_{H}(S)$ and that $e$ is distinguished in Lie $C_{H}(S)$, cf. Remark 2.10(ii). Thus, by the distinguished case just proved, $\lambda$ is a cocharacter of $C_{H}(S)$ associated to $e \in$ $\mathfrak{c}_{\mathfrak{h}}(S)=\operatorname{Lie} C_{H}(S)$. Observe that $\operatorname{dim} S=\operatorname{rank} C_{C_{H}(S)}(e)=\operatorname{rank} C_{H}(e)$. It thus follows from Lemma 3.3 applied to $C_{H}(S) \leq H$ that $\lambda$ is a cocharacter of $H$ associated to $e$, as claimed.

Our next result shows that the forward inclusion of $(\dagger)$ holds provided there is at least one cocharacter of $G$ that is associated to $e \in \mathcal{N} \cap \mathfrak{h}$ and takes values in $H$.

Lemma 3.17. Let $e \in \mathfrak{h} \cap \mathcal{N}$. Then $\Omega_{H}^{a}(e) \subseteq \Omega_{G}^{a}(e) \cap Y(H)$, provided $\Omega_{G}^{a}(e) \cap Y(H)$ is non-empty.

Proof. Let $\lambda \in \Omega_{G}^{a}(e) \cap Y(H)$ and let $\mu \in \Omega_{H}^{a}(e)$. Then, by Proposition 3.16, $\lambda$ is a cocharacter of $H$ associated to $e$. So, by Proposition 2.11(ii), there exists an $x \in C_{H}(e)^{\circ}$ such that $x \lambda x^{-1}=\mu$. Clearly, $C_{H}(e)^{\circ} \leq C_{G}(e)^{\circ}$. By Remark 2.10(i) it follows that $\mu$ is thus a cocharacter of $G$ associated to $e$. 
Combining Proposition 3.16 and Lemma 3.17 gives our next result.

Theorem 3.18. Let $e \in \mathfrak{h} \cap \mathcal{N}$. If $\Omega_{G}^{a}(e) \cap Y(H)$ is non-empty, then $\Omega_{H}^{a}(e)=\Omega_{G}^{a}(e) \cap Y(H)$.

Let $S$ be a linearly reductive group acting on $G$ by automorphisms, so that $G$ is an $S$-group. Set $H=C_{G}(S)^{\circ}$ which is reductive, by Proposition 2.2(i). Let $e \in \mathfrak{h}=\mathfrak{c}_{\mathfrak{g}}(S)$ be nilpotent. In the proof of [17, Thm. C], R. W. Richardson showed that there always exists a cocharacter of $G$ which belongs to the optimal class $\Omega(e)$ defined by $e$ so that its image lies in $H$, see $[17, \S 8]$. For our purpose we need a variant of this result: we require the existence of a cocharacter of $G$ which is associated to $e$ (rather than merely being optimal) so that its image lies in $H$. That is, we need to construct an $S$-fixed cocharacter of $G$ which is associated to $e$. This is done in Lemma 3.20 with the aid of Richardson's result Proposition 2.3. For our next result, recall the subgroup $Q_{e}$ of $N_{e}$ from (2.18).

Lemma 3.19. Let $e \in \mathfrak{h}=\mathfrak{c}_{\mathfrak{g}}(S)$ be nilpotent. Then

(i) $\Omega^{a}(e)$ is S-stable;

(ii) $C_{G}(e)$ is S-stable;

(iii) $Q_{e}$ is $S$-stable.

Proof. (i) Let $\lambda \in \Omega^{a}(e)$. Since the induced actions of $S$ and $G$ on $\mathfrak{g}$ are compatible in the sense of (2.1) and linear, we have

$$
\operatorname{Ad}((s \cdot \lambda)(t)) e=s \cdot \operatorname{Ad}(\lambda(t)) e=s \cdot\left(t^{2} e\right)=t^{2} e,
$$

for every $s \in S$ and $t \in k^{*}$; so $e \in \mathfrak{g}(2, s \cdot \lambda)$ for every $s \in S$. Clearly, if $e$ is distinguished in Lie $L$, then $e=s \cdot e$ is distinguished in $\operatorname{Lie}(s \cdot L)$ and $s \cdot L$ is another Levi subgroup of $G$ for $s \in S$. Finally, $\lambda\left(k^{*}\right) \leq \mathcal{D} L$ implies that $(s \cdot \lambda)\left(k^{*}\right) \leq s \cdot \mathcal{D} L=\mathcal{D}(s \cdot L)$ for $s \in S$. It follows that $s \cdot \lambda \in \Omega^{a}(e)$ for any $s \in S$.

(ii) Again, by the compatibility of the actions of $S$ and $G$ on $\mathfrak{g}$, we obtain

$$
\operatorname{Ad}(s \cdot g) e=s \cdot \operatorname{Ad}(g) e=s \cdot e=e,
$$

for any $g \in C_{G}(e), s \in S$.

(iii) Since $C_{G}(e)$ is $S$-stable, so is $R_{e}=R_{u}\left(C_{G}(e)\right)$. Since $\Omega^{a}(e)$ is $S$ stable, by part (i), it follows from Corollary 2.16 that $Q_{e}$ is also $S$-stable. 
Lemma 3.20. Let $e \in \mathfrak{h}=\mathfrak{c}_{\mathfrak{g}}(S)$ be nilpotent. Then the following hold.

(i) There exists an $S$-stable Levi subgroup $\lambda\left(k^{*}\right)$ of $Q_{e}$.

(ii) There exists an $S$-fixed cocharacter in $\Omega^{a}(e)$.

(iii) There exists an $S$-stable Levi subgroup $C_{G}(e, \lambda)$ of $C_{G}(e)$.

Proof. (i) Let $\lambda^{\prime} \in \Omega^{a}(e)$ so that $Q_{e}=\lambda^{\prime}\left(k^{*}\right) R_{e}$. Then $R_{u}\left(Q_{e}\right)=R_{e}$. According to Corollary 2.16, $R_{e}$ acts simply transitively on $\Omega^{a}(e)$. Thus $R_{e}$ acts simply transitively on the set of Levi subgroups of $Q_{e}$, Remark 2.17. Thanks to Lemma 3.19(iii), $Q_{e}$ is $S$-stable. The desired result now follows from Proposition 2.3.

(ii) Let $\lambda\left(k^{*}\right)$ be as in (i), i.e., $(s \cdot \lambda)\left(k^{*}\right)=s \cdot\left(\lambda\left(k^{*}\right)\right)=\lambda\left(k^{*}\right)$ for every $s \in S$. Since $e \in \mathfrak{g}(2, \lambda) \cap \mathfrak{g}(2, s \cdot \lambda)$, it follows from [7, Lem. 4.11] that $s \cdot \lambda=\lambda$ for every $s \in S$.

(iii) Let $\lambda$ in $\Omega^{a}(e)$ be $S$-fixed as in (ii). Since $S$ and $G$ act compatibly on $Y(G)$ in the sense of $(2.1), C_{G}(\lambda)$ is $S$-stable. For,

$$
(s \cdot g) \cdot \lambda(t)=s \cdot(g \cdot \lambda)(t)=s \cdot \lambda(t)=\lambda(t)
$$

for all $s \in S, g \in C_{G}(\lambda)$, and $t \in k^{*}$. Consequently, since $C_{G}(e)$ is $S$-stable, by Lemma 3.19 (ii), so is $C(e, \lambda)=C_{G}(e) \cap C_{G}(\lambda)$. The result now follows from Proposition 2.15.

Finally, Theorem 1.2 follows from Theorem 3.18 and Lemma 3.20(ii):

THEOREM 3.21. Let $S$ be a linearly reductive group acting on $G$ by automorphisms and set $H=C_{G}(S)^{\circ}$. Let $e \in \mathfrak{h} \cap \mathcal{N}$. Then $\Omega_{H}^{a}(e)=$ $\Omega_{G}^{a}(e) \cap Y(H)$.

We record various special cases of Theorem 3.21 as separate corollaries.

Since a Levi subgroup of $G$ is of the form $C_{G}(S)$ for some torus $S$ of $G$, our next result is immediate from Theorem 3.21.

Corollary 3.22. Let $H$ be a Levi subgroup of $G$. Let $e \in \mathfrak{h} \cap \mathcal{N}$. Then $\Omega_{H}^{a}(e)=\Omega_{G}^{a}(e) \cap Y(H)$.

Likewise, our next result is immediate from Theorem 3.21 and Remark 2.5. 
Corollary 3.23. Let $\sigma$ be a semisimple automorphism of $G$. Let $H=$ $C_{G}(\sigma)^{\circ}$. Let $e \in \mathfrak{h} \cap \mathcal{N}$. Then $\Omega_{H}^{a}(e)=\Omega_{G}^{a}(e) \cap Y(H)$.

Here is a special case of Corollary 3.23. Assume that $G$ is simple and let $\gamma$ be a non-trivial graph automorphism of $G$. If char $k$ is coprime to the order of $\gamma$, then $\gamma$ is a semisimple automorphism of $G$.

Corollary 3.24. Let $G$ be simple and let $\gamma$ be a non-trivial graph automorphism of $G$. Suppose that char $k$ is coprime to the order of $\gamma$. Let $H=C_{G}(\gamma)^{\circ}$. Let $e \in \mathfrak{h} \cap \mathcal{N}$. Then $\Omega_{H}^{a}(e)=\Omega_{G}^{a}(e) \cap Y(H)$.

We give two examples for Corollary 3.24.

EXAmple 3.25. Suppose that $p \neq 2$. Let $V$ be a finite-dimensional $k$-vector space. Let $H$ be either $\operatorname{Sp}(V)$ or $\mathrm{SO}(V)$. Observe that $H$ is the fixed point subgroup of an involution of $\mathrm{SL}(V)$ (cf. [24, $§ 11$ p. 169]) and thus Corollary 3.24 applies. That is, the cocharacters of the classical groups $H=\mathrm{Sp}(V)$ or $\mathrm{SO}(V)$ associated to a given nilpotent element $e$ in the Lie algebra of $H$ are precisely the cocharacters of the ambient linear group $\mathrm{SL}(V)$ associated to $e$ whose image lies in $H$.

ExAmple 3.26. Suppose that char $k>3$. Let $G$ be of type $D_{4}$ and let $\gamma$ be the triality graph automorphism of $G$. Then $H=C_{G}(\gamma)^{\circ}$ is of type $G_{2}$ and so Corollary 3.24 applies. Thus, for a given nilpotent element $e \in \mathfrak{h}$ we can realize the cocharacters of $H$ associated to $e$ as the cocharacters of $G$ associated to $e$ that are $\gamma$-fixed.

Recall from subsection 2.3 that for $s \in G$ semisimple, $C_{G}(s)^{\circ}$ is a pseudo-Levi subgroup of $G$. The next result is again a special case of Corollary 3.23 .

Corollary 3.27. Let $H$ be a pseudo-Levi subgroup of $G$. Let $e \in$ $\mathfrak{h} \cap \mathcal{N}$. Then $\Omega_{H}^{a}(e)=\Omega_{G}^{a}(e) \cap Y(H)$.

Clearly, each pseudo-Levi subgroup of $G$ is of maximal rank. The subsystems corresponding to maximal rank, semisimple subgroups of a simple group $G$ are determined by means of the algorithm of Borel and de Siebenthal [3], see also [4, Ex. Ch. VI §4.4]. Using Corollary 3.27, the algorithm of Borel and de Siebenthal, as well as Deriziotis' characterization of maximal rank reductive subgroups (cf. [6, §2.15]), we can generalize Corollary 3.27 to arbitrary maximal rank reductive subgroups.

Our next result is Theorem 1.1. 
THEOREM 3.28. Let $H$ be a connected reductive subgroup of $G$ of maximal rank. Let $e \in \mathfrak{h} \cap \mathcal{N}$. Then $\Omega_{H}^{a}(e)=\Omega_{G}^{a}(e) \cap Y(H)$.

Proof. Thanks to Lemma 2.19 and by passing to simple factors, we may assume that $G$ is simple. Let $H$ be a maximal rank reductive subgroup of $G$. Let $T$ be a maximal torus of $G$ contained in $H$. Let $\Pi$ be a set of simple roots of $\Psi=\Psi(G, T)$, let $\varrho$ be the highest root of $\Psi^{+}$, and let $W$ be the Weyl group of $G$ with respect to $T$. Let $\Phi=\Phi(H, T)$ be the root system of $H$; in particular $\Phi$ is a semisimple subsystem of $\Psi$. Since char $k$ is good for $G$, it follows from Deriziotis' Criterion (cf. $[6, \S 2.15]$ ) that $H$ is of the form $H=C_{G}(s)^{\circ}$ if and only if $\Phi$ admits a base which is $W$-conjugate to a proper subset of $\Pi \cup\{-\varrho\}$. This construction coincides with the inductive step in the Borel-de Siebenthal procedure, [3]. Thanks to [12, Prop. 16], since $p$ is good for $G$, it is also good for $H$. Because every maximal rank subsystem of $\Psi$ is obtained by an iteration of the Borel-de Siebenthal procedure, the proposition follows by a repeated application of this algorithm, Deriziotis' Criterion, and Corollary 3.27.

THEOREM 3.29. Let $H$ be a connected regular reductive subgroup of $G$. Let $e \in \mathfrak{h} \cap \mathcal{N}$. Then $\Omega_{H}^{a}(e)=\Omega_{G}^{a}(e) \cap Y(H)$.

Proof. Let $T$ be a maximal torus of $G$ normalizing $H$. Then $T H$ is a connected reductive subgroup of $G$ of maximal rank. Since $\mathcal{D}(T H)=\mathcal{D} H$ is the semisimple part of $H$ and so $T H=Z(T H) \mathcal{D} H$, the result follows from Lemma 2.19 and Theorem 3.28.

Our next two examples show that by iterating our results we can ensure that $(\dagger)$ holds even in cases where we cannot apply Theorem 3.21 directly.

ExAmple 3.30. Let $G$ be of type $F_{4}$ and let $K$ be a connected simple subgroup of $G$ of type $D_{4}$ and $H$ a connected subgroup of $K$ of type $G_{2}$. Suppose that $p$ is good for $G$. Then a successive application of Theorem 3.28 and the conclusion from Example 3.26 show that $(\dagger)$ also holds for the embedding $H \leq G$. Note that $H$ is not a regular subgroup of $G$ so that we cannot invoke Theorem 3.29 directly to the embedding $H \leq G$. This is the standard embedding of $G_{2}$ in $F_{4}$. We discuss a different embedding of $G_{2}$ in $F_{4}$ in characteristic 7 in Example 3.33 below. 
ExAmple 3.31. Let $G$ be of type $E_{8}$ and suppose that $p$ is good for $G$. Let $K$ be a maximal rank subgroup of $G$ of type $D_{4} \times D_{4}$, and let $H$ be a connected subgroup of $K$ of type $D_{4}$ embedded diagonally into $K$. Then $H$ is the fixed point subgroup of the involution interchanging the $D_{4}$-factors in $K$. Note that $H$ is not a regular subgroup of $G$, so we cannot invoke Theorem 3.29. Nevertheless, it follows from Corollary 3.23 and Theorem 3.28 that $(\dagger)$ holds for the embedding $H \leq G$.

Remarks 3.32. (i) Theorem 3.28 answers a question posed by J. C. Jantzen, cf. [7, §5.12].

(ii) The forward inclusion of Corollary 3.27 was already proved in [12, Prop. 23, Rem. 25] by different methods.

EXAmple 3.33. In [26, Thm. 1(c)], D. Testerman showed that there is a maximal subgroup of type $G_{2}$ in $F_{4}$ in characteristic $p=7$; see also [19, Thm. 1]. In this case let $G$ be the ambient group of type $F_{4}$ and let $H$ be the maximal subgroup of type $G_{2}$. The fusion of the unipotent classes of this embedding has been determined by R. Lawther (unpublished). Since $p=7$ is good for $G$, this also determines the fusion of the nilpotent classes of $\mathfrak{h}$ in $\mathfrak{g}$; this is given in terms of the corresponding Bala-Carter labellings as follows:

\begin{tabular}{|c|ccccc|}
\hline$G_{2}$ & 1 & $A_{1}$ & $\tilde{A}_{1}$ & $G_{2}\left(a_{1}\right)$ & $G_{2}$ \\
\hline$F_{4}$ & 1 & $A_{1} \tilde{A}_{1}$ & $\tilde{A}_{2} A_{1}$ & $F_{4}\left(a_{3}\right)$ & $F_{4}\left(a_{2}\right)$ \\
\hline
\end{tabular}

Table 1: The fusion of nilpotent classes for $G_{2} \leq_{\max } F_{4}(p=7)$.

In contrast to the standard (non-maximal) embedding of $G_{2}$ in $F_{4}$ which exists in any characteristic (Example 3.30), we cannot deduce $(\dagger$ ) for the embedding $H \leq G$ directly or by iterating our results, as $H$ is maximal in $G$. Nevertheless, our methods allow us to easily deduce that $(\dagger)$ holds for all but one of the nilpotent classes of $\mathfrak{h}$.

Note that the trivial cocharacter is associated to $e=0$ for both $H$ and $G$. If $e$ belongs to the regular or subregular class in $\mathfrak{h}$, then according to Table 1 and $[5, \S 5.9]$, the corresponding $G$-classes $F_{4}\left(a_{3}\right)$ and $F_{4}\left(a_{2}\right)$ in $\mathcal{N}$ are distinguished. Therefore, the result follows by Lemma 3.1. Let $e$ belong to the $G_{2}$-class with label $\tilde{A}_{1}$. According to Table 1 and $[5, \mathrm{Ch} .13]$, the 
reductive parts of the centralizers $C_{H}(e)$ and $C_{G}(e)$ of this class in $G_{2}$ and the corresponding class $\tilde{A}_{2} A_{1}$ in $F_{4}$ are both of type $A_{1}$. In particular, $C_{H}(e)$ and $C_{G}(e)$ have the same rank and Theorem 3.14 gives the desired result in this case.

For the remaining pair of nilpotent orbits one can use Theorem 3.18 and show directly that $(\dagger)$ also holds in this case. Fix maximal tori $T_{H}$ and $T$ of $H$ and $G$ respectively, so that $T_{H} \leq T$. Let $\alpha$ be the long simple root of $H$ with respect to $T_{H}$ and let $\left\{\alpha_{1}, \ldots, \alpha_{4}\right\}$ be the set of simple roots of $G$ with respect to $T$ so that $\alpha_{1}$ and $\alpha_{2}$ are long. Let $e=e_{\alpha}$, a non-trivial root vector in the root space of $\alpha$ in $\mathfrak{g}$. Then $e$ belongs to the $H$-class in $\mathfrak{h}$ with label $A_{1}$ and the coroot $\alpha^{\vee} \in Y(H)$ is an associated cocharacter of $e$, see $[7, \S 5.13]$. Let $L_{\left\{\alpha_{1}, \alpha_{3}\right\}}$ be the standard Levi subgroup of $G$ of type $A_{1} \tilde{A}_{1}$ with root system $\left\{ \pm \alpha_{1}, \pm \alpha_{3}\right\}$. Let $M=s_{\alpha_{2}}\left(L_{\left\{\alpha_{1}, \alpha_{3}\right\}}\right)$, where $s_{\alpha_{2}}$ is the simple reflection in the Weyl group of $G$ corresponding to $\alpha_{2}$. Then $M$ is another Levi subgroup of $G$. Using Lawther's explicit fusion calculations, one can show that $\alpha^{\vee}\left(k^{*}\right) \leq \mathcal{D} M$ and that $e$ is distinguished in $\mathfrak{m}$. Finally, since $e \in \mathfrak{h}\left(2, \alpha^{\vee}\right) \subset \mathfrak{g}\left(2, \alpha^{\vee}\right)$, it follows that $\alpha^{\vee} \in \Omega_{G}^{a}(e)$. Therefore, $\alpha^{\vee} \in \Omega_{G}^{a}(e) \cap Y(H)$, and consequently, by Theorem 3.18, we have $\Omega_{H}^{a}(e)=\Omega_{G}^{a}(e) \cap Y(H)$.

Acknowledgements. The first author acknowledges funding by the EPSRC. We are grateful to R. Lawther for making available information on the fusion of the nilpotent classes given in Table 1 in Example 3.33 from an unpublished manuscript. Further, we would like to thank S. Goodwin for carefully reading earlier versions of the paper and for suggesting various improvements. We are particularly indebted to G. McNinch for very helpful discussions and comments.

\section{REFERENCES}

[1] P. Bardsley and R. W. Richardson, Étale slices for algebraic transformation groups in characteristic p, Proc. London Math. Soc. (3), 51 (1985), no. 2, 295-317.

[2] A. Borel, Linear Algebraic Groups, Graduate Texts in Mathematics, 126, SpringerVerlag, 1991.

[3] A. Borel and J. de Siebenthal, Les sous-groupes fermés de rang maximum des groupes de Lie clos, Comment. Math. Helvet., 23 (1949), 200-221.

[4] N. Bourbaki, Groupes et algèbres de Lie, Chapitres 4, 5 et 6, Hermann, Paris, 1975.

[5] R. W. Carter, Finite groups of Lie type. Conjugacy classes and complex characters, Pure and Applied Mathematics, New York, 1985. 
[6] J. E. Humphreys, Conjugacy classes in semisimple algebraic groups, Mathematical Surveys and Monographs, 43, American Mathematical Society, Providence, RI, 1995.

[7] J. C. Jantzen, Nilpotent Orbits in Representation Theory, Lie Theory, Lie Algebras and Representations (J.-P. Anker and B. Orsted, eds.), Progress in Math., vol. 228, Birkhäuser Boston, 2004.

[8] G. R. Kempf, Instability in Invariant Theory, Ann. Math., 108 (1978), 299-316.

[9] R. Lawther, Jordan block sizes of unipotent elements in exceptional algebraic groups, Comm. Algebra, 23 (1995), no. 11, 4125-4156.

[10] G. McNinch, Nilpotent orbits over ground fields of good characteristic, Math. Ann., 329 (2004), no. 1, 49-85.

[11] Optimal SL(2)-homomorphisms, Comment. Math. Helv., 80 (2005), no. 2, 391-426.

[12] G. McNinch and E. Sommers, Component groups of unipotent centralizers in good characteristic, Special issue celebrating the 80th birthday of Robert Steinberg, J. Algebra, 260 (2003), no. 1, 323-337.

[13] G. D. Mostow, Fully reducible subgroups of algebraic groups, Amer. Math. J., 78 (1956), 200-221.

[14] M. Nagata, Complete reducibility of rational representations of a matric group, J. Math. Kyoto University, 1 (1961), 87-99.

[15] A. Premet, An analogue of the Jacobson-Morozov Theorem for Lie algebras of reductive groups of good characteristics, Trans. Amer. Math. Soc., 347 (1995), 2961-2988.

[16] - Nilpotent orbits in good characteristic and the Kempf-Rousseau theory, Special issue celebrating the 80th birthday of Robert Steinberg, J. Algebra, 260 (2003), no. 1, 338-366.

[17] R. W. Richardson, On orbits of algebraic groups and Lie groups, Bull. Austral. Math. Soc., 25 (1982), no. 1, 1-28.

[18] G. Rousseau, Immeubles sphériques et théorie des invariants, C. R. Acad. Sci. Paris, 286 (1987), 247-250.

[19] G. M. Seitz, Maximal subgroups of exceptional algebraic groups, Mem. Amer. Math. Soc., 441, 1991.

[20] Unipotent elements, tilting modules, and saturation, Invent. Math., 141 (2000), no. 3, 467-502.

[21] P. Slodowy, Theorie der optimalen Einparameteruntergruppen, Algebraische Transformationsgruppen und Invariantentheorie (H. Kraft, P. Slodowy and T. A. Springer, eds.), DMV Seminar, 13, Birkhäuser Verlag, Basel, 1989.

[22] T. A. Springer, Linear Algebraic Groups, Second edition, Progress in Mathematics, 9, Birkhäuser Boston, Inc., Boston, MA, 1998.

[23] R. Steinberg, Endomorphisms of Linear Algebraic Groups, Mem. Amer. Math. Soc., 80, 1968 .

[24] Notes on Chevalley Groups, Yale University, New Haven (1968).

[25] T. A. Springer and R. Steinberg, Conjugacy classes, Seminar on algebraic groups and related finite groups, Lecture Notes in Mathematics, 131, Springer-Verlag, Heidelberg, 1970, pp. 167-266. 
[26] D. M. Testerman, A construction of certain maximal subgroups of the algebraic groups $E_{6}$ and $F_{4}, \mathrm{~J}$. Algebra, 122 (1989), no. 2, 299-322.

Russell Fowler

School of Mathematics

University of Birmingham

Birmingham, B15 2TT

UK

Gerhard Röhrle

Fakultät für Mathematik

Ruhr-Universität Bochum

D-44780 Bochum

Germany

gerhard.roehrle@rub.de 